\title{
Small Satellites
}

\section{By STEVEN GAO, Senior Member IEEE}

Guest Editor

\section{MARTIN N. SWEETING, Member IEEE}

Guest Editor

\section{SHINICHI NAKASUKA}

Guest Editor

\section{SIMON PETER WORDEN}

Guest Editor

\section{INTRODUCTION}

Small satellite is a disruptive technology in space industries. Traditionally, space industries were dominated by satellites which have thousands of kilograms and are bulky and expensive. Small satellites denote a new generation of miniaturized satellites which, by taking advantages of modern technologies (e.g., integrated circuits, digital signal processing, MEMS, and additive manufacturing), can achieve a significant reduction in volume, mass, development time, and cost of satellites. During recent decades, small satellites, including CubeSats, NanoSats, MiniSats, and MicroSats, have undergone rapid developments, and are playing an increasingly larger role in exploration, technology demonstration, scientific research, and education. These miniature satellites provide a low-cost platform for missions, including planetary space exploration, Earth observations, fundamental Earth and space science, and developing precursor science instruments like laser communications and millimeterwave communications for intersatellite and intrasatellite links, and autonomous movement capabilities. They also allow educators an inexpensive means to engage students in all phases of satellite development, operation, and exploitation through real-world, hands-on research and development experience on rideshare launch opportunities. A number of miniaturized satellites can form spaceborne wireless sensor networks in the space, which are also going to play an important role in Internet of Space (IoS) of the future.

This special issue covers the most recent developments in small satellites. This special issue includes 11 invited papers covering different aspects of small satellites, spanning topics from a comprehensive review of small satellite development, to advanced technologies such as propulsion technology, attitude

Digital Object Identifier: 10.1109/JPROC.2018.2805267 control thruster, advanced antennas for wireless systems, radar technology, advanced energy storage systems, robotics and autonomous system (RAS) technologies, satellite formation flying, onboard processing, deployable structures, and science applications such as thermospheric variations from global navigation satellite systems (GNSSs) and accelerometer measurements on small satellites.

\section{OVERVIEW OF THE SPECIAL ISSUE}

The issue starts with the review paper "Modern Small Satellites-Changing the Economics of Space" by Sweeting. This paper reviews the history of small satellite development and then summarizes their present capabilities and applications. This is followed by a look at the future technology trends that small satellites can exploit-both in Earth orbit and for exploration of the solar system. The paper also includes many examples of different types of small satellites.

The paper "Space Propulsion Technology for Small Spacecraft” by Krejci and Lozano discusses the different propulsion principles applicable to small satellites, and presents a classification of available propulsion solutions, including a variety of different chemical and EP systems of varying 
complexity and performance. A discussion of the predominant scaling laws for miniaturization of different propulsion systems is given, identifying the limiting factors in miniaturization on thruster, and system level is given. Based on this, a section discussing the propulsion related requirements and state-of-the-art technologies for the extreme cases of chip and membrane satellites, and infinite specific impulse missions are discussed.

The paper "Development and Testing of a 3-D-Printed Cold Gas Thruster for an Interplanetary CubeSat" by Lightsey et al. describes the development and testing of a cold gas attitude control thruster produced for the BioSentinel spacecraft, a CubeSat that will operate beyond Earth orbit. The thruster will reduce the spacecraft rotational velocity after deployment, and for the remainder of the mission it will periodically unload momentum from the reaction wheels. The design, development, and test campaign for the thruster system are presented.

The paper "Advanced Antennas for Small Satellites" by Gao et al. presents a comprehensive review of recent development in antennas for wireless systems onboard small satellites (MiniSats, MicroSats, NanoSats, CubeSats). Antenna is one of the key components onboard small satellites as its design determines the performance of all the wireless systems including telemetry, tracking and control, high-speed data downlink, navigation, intersatellite communications, intrasatellite communications, wireless power transfer, radars and sensors, etc. Many recent examples of advanced antennas for small satellite applications are shown and discussed. A conclusion and future development in antennas for small satellites are given in the end.

The paper "Radar Technologies for Earth Remote Sensing from CubeSat Platforms" by Peral et al. reviews the state of the art and future developments of CubeSat radar missions for Earth remote sensing and the implications for NASA's current and future
Earth Science program. Space-based radar observations have transformed our understanding of Earth over the last several decades. The key enabling technologies for RF, digital and antennas are surveyed, as well as the evolution of the CubeSat avionics, in the aspects that mostly impact radar development, namely power, volume, and attitude control and knowledge and precision orbit determination (POD). Various radar applications that could benefit from low-cost CubeSat platforms, such as altimetry, sounding, precipitation profiling, scatterometry, synthetic aperture radar (SAR), and interferometric SAR (InSAR) are discussed.

The paper "Energy Storage Technologies for Small Satellite Applications" by Chin et al. provides a general review of performance capabilities of state-of-the-art lithium-ion battery technologies. With advances in CubeSat technologies, the power and energy demands have also increased dramatically, necessitating the need for larger deployable solar arrays, lower power electronics, efficient energy storage systems, and even energy transfer/harvesting systems. In terms of energy storage, more advanced battery chemistries with higher energy densities and higher power capabilities over a wider operating temperature range are also a fundamental need. Other advanced energy storage systems for small satellite applications are also discussed.

The paper "Robotics and AIEnabled On-Orbit Operations With Future Generation of Small Satellites" by Nanjangud et al. provides an overview of the robotics and autonomous system (RAS) technologies that enable robotic on-orbit operations (O3) on SmallSat platforms. The low cost and short lead time of small satellites have led to their use in science-based missions, Earth observation, and interplanetary missions. They are also key instruments in orchestrating technological demonstrations for $\mathrm{O} 3$ such as inspection and spacecraft servicing with planned roles in active debris removal and on-orbit assembly. Major RAS topics such as sensing and perception; guidance, navigation \& control (GN\&C) microgravity mobility and mobile manipulation; and autonomy are discussed from the perspective of relevant past and planned missions.

The paper "A Survey on Formation Control of Small Satellites" by Liu and Zhang comprehensively reviews the state-of-the-art development in formation control of small satellites, including satellite formation flying, distributed satellite systems, and fractionated satellite formation. Various formation control architectures and methods of small satellites are introduced. Coordinative control of multiple small satellites is also reviewed, covering coordinative control of satellite formation, coordinative attitude control of satellite formation, and coordinative coupled attitude and orbit control of satellite formation.

The paper "Onboard Processing With Hybrid and Reconfigurable Computing on Small Satellites" by George and Wilson surveys the challenges and opportunities of onboard computers for small satellites and focuses upon new concepts, methods, and technologies that are revolutionizing their capabilities, in terms of two guiding themes: hybrid computing and reconfigurable computing. Due to the increasing demands of onboard sensor and autonomous processing, one of the principal needs and challenges for future spacecraft is onboard computing. With new technologies, such as CHREC Space Processor (CSP), it demonstrates how system designers can exploit hybrid and reconfigurable computing on small satellites to harness these advantages for a variety of purposes, and it highlight several recent missions by NASA and industry that feature these principles and technologies.

The paper "Deployable Techniques for Small Satellites" by Miyazaki provides an overview of the past and current research and development of a deployable structure for small satellites, and discusses the future of a 
deployable structure for small satellites. A deployable structure serves an important function in small satellites as their performance is improved steadily. Larger deployable solar array paddles are expected as the satellite requires a larger amount of electric power. The synthetic aperture radar (SAR) system for small satellites requires a deployable antenna. Many examples of deployable structures are discussed.

The paper "Thermospheric Variations From GNSS and Accelerometer Measurements on Small Satellites" by
Jin et al. presents an overview of past and present developments and efforts in sensing and modeling thermospheric density and wind variations are presented, as well as the future challenges and perspectives for GNSS and accelerometers on small satellites. Monitoring and understanding geophysical processes in the thermosphere is primordial for space physics, low Earth orbiters, and groundbased technologies. GNSS and accelerometers on small satellites can sense neutral density and wind variations with unprecedented accuracy, which contribute to understanding of thermospheric variations and improving the current empirical and physical models.

\section{Acknowledgement}

The Guest Editors would like to thank all authors and reviewers for their contributions to this Special Issue. This issue would not have been possible without the support from Prof. S. E. Barbin, University of Sao Paulo, Brazil. They would also like to thank the editorial team, in particular V. Damle, J. Sun, and H. J. Trussell, for their support.

\section{ABOUT THE GUEST EDITORS}

Steven Gao (Senior Member, IEEE) received the Ph.D. degree from Shanghai University, China.

$\mathrm{He}$ is a Professor and Chair of RF and Microwave Engineering, and the Director of Postgraduate Research at the School of Engineering and Digital Arts, University of Kent, Canterbury, U.K. He coedited/coauthored two books including Space Antenna Handbook (New York, NY, USA: Wiley, 2012) and Circularly Polarized Antennas (New York, NY,

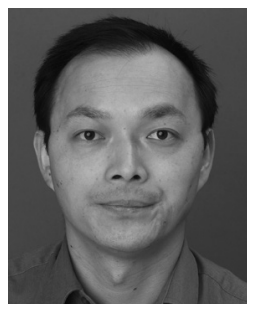

USA: Wiley-IEEE, 2014), over 300 papers and four patents. His main areas of expertise are in smart antenna, phased array, small antennas, MIMO, broadband and multiband antennas, RF front ends, FSS, and their applications into satellite communication, small satellites, $5 \mathrm{G}$ mobile communications, and radars.

Prof. Gao is an Associate Editor of four journals including the IEEE Transactions on Antennas and Propagation, Radio Science, ieEe Access, and IET Circuit, Devices and Systems; and the Editor-in-Chief for Wiley Book Series on "Microwave and Wireless Technologies." He was a Distinguished Lecturer of the IEEE Antennas and Propagation Society (2014-2016), General Chair of the 2013 Loughborough Antenna and Propagation Conference, a Guest Editor of the IEEE Transactions on Antennas And Propagation Special Issue on “Antennas for Satellite Communication” (2015), and a Guest Editor of the IET Circuits, Devices \& Systems Special Issue on "Photonic and RF Communications Systems" (2014). He is a member of the editorial boards of the International Journal of Space Science and Engineering, the International Journal of RF and Microwave ComputerAided Engineering, IET Circuits, Devices and Systems, Chinese Journal of Electronics, Chinese Journal of Radio Science, etc. He was a Plenary Speaker at AES'2014, an Invited Speaker at IWA'2017 (Greece), UCMM'2017 (U.K.), IWAT'201 (Sydney, 2014), SOMIRES'2013 (Japan, 2013), APCAP'2014 (China, 2014), etc. He is a Fellow of the Institution of Engineering and Technology (IET), U.K., and the Royal Aeronautical Society, U.K. He received the 2017 CST University Publication Award for a paper in the IEEE Transactions on Antennas and Propagation, and the 2016 IET Premium Award for the Best Paper in IET Microwave, Antennas and Propagation.

Martin N. Sweeting (Member, IEEE) received the B.Sc. and Ph.D. degrees in electronic engineering from the University of Surrey, Guildford, U.K.

In 1985, he founded a spinoff University company (SSTL), Guildford, U.K., which has since designed, built, launched, and operated in orbit 54 nanosatellites, microsatellites, and minisatellites, including the international Disaster Monitoring Constellation (DMC) and additionally the 34

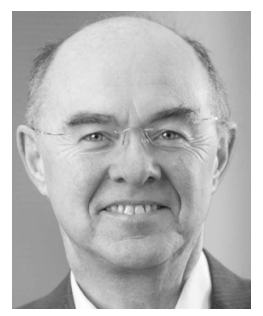

navigation payloads for the European Galileo operational constellation. $\mathrm{He}$ is Executive Chairman of SSTL with 550 staff and a distinguished professor at the University of Surrey leading the academic Surrey Space Centre researching advanced small satellite concepts and techniques.

Sir Martin has been appointed OBE (Officer of the British Empire) and knighted by HM The Queen, and has received the prestigious von Karman Wings Award from CalTech/JPL in the United States. In 2014, he received the Chinese Academy of Sciences/COSPAR Jeoujang Jaw Award recognizing his contribution to international space development. In 2016, he was identified as one of the U.K.'s 20 most influential engineers. In 2017, he was listed as one of the 500 most influential people in the United Kingdom. He is a Fellow of the Royal Society (the U.K. Academy of Sciences) and a Fellow of the Royal Academy of Engineering, who has pioneered rapidresponse, low-cost and highly capable small satellites utilizing modern consumer (COTS) electronics devices to change the economics of space.

Shinichi Nakasuka graduated from the University of Tokyo, Tokyo, Japan, in 1983 and received the Ph.D. degree from the University of Tokyo, in 1988.

He joined IBM Research during 1988-1990. Then, he was working for the Department of Aeronautics and Astronautics, University of Tokyo, as a Lecturer in 1990 and an Associate Professor in 1993, where he has been a Professor since 2004. His

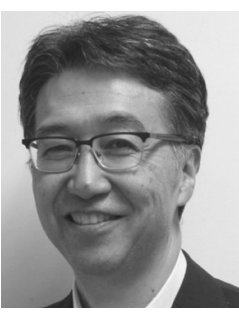
research interests include micro/nano/picosatellites, navigation/guidance/ control of spacecraft, novel space systems, autonomy and intelligence for space systems. He developed and launched the world first CubeSat "XI-IV" in 2003, and since then has successfully launched eight micro/nano/picosatellites for Earth observation, communication, and deep space missions.

Prof. Nakasuka is a member of the Japan Society for Aeronautical and Space Sciences (JSASS), the Society of Instrument and Control Engineers (SICE), and the International Academy of Astronautics (IAA), and was the Chairperson of the IFAC Aerospace Technical Committee. He has been a member of the Space Policy Committee of the Japanese Cabinet Office since 2012.

Simon Peter "Pete" Worden was born in 1949 in Michigan, USA. He received the B.Sc. degree in physics and astronomy from the University of Michigan, Ann Arbor, MI, USA and the Ph.D. degree in astronomy for the University of Arizona, Tucson, AZ, USA.

$\mathrm{He}$ is the Chairman of the Breakthrough Prize Foundation and Executive Director of the foundation's “Breakthrough Initiatives." Prior to joining the Breakthrough Prize Foundation, he was

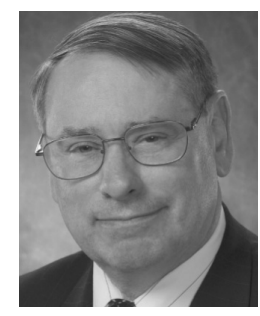


Director of NASA's Ames Research Center at Moffett Field, CA, USA, until his retirement on March 31, 2015. He has held several positions in the U.S. Air Force and was Research Professor of Astronomy at the University of Arizona. He is a recognized expert on space and science issues, both civil and military, and has been a leader in building partnerships between governments and the private sector internationally. He has authored or coauthored more than 150 scientific papers in astrophysics space sciences, and strategic studies. He served as a scientific coinvestigator for three NASA space science missions, most recently the Interface Region Imaging Spectrograph launched in 2013 to study the Sun.

Dr. Worden received the NASA Outstanding Leadership Medal for the 1994 Clementine Mission to the moon. He was named the 2009
Federal Laboratory Consortium Laboratory Director of the Year and is the recipient of the 2010 Arthur C. Clarke Innovator's Award. On July 20, 2015 at the Royal Society in London, U.K., Yuri Milner and Stephen Hawking launched the Breakthrough Initiatives. At the press conference, Dr. Worden was introduced as the Chairman for the Breakthrough Prize Foundation. In this capacity he leads the Breakthrough Initiatives. On April 12, 2016 in the One World Observatory in New York, NY, USA, Yuri Milner and Stephen Hawking announced the Breakthrough StarShot Initiative to develop and launch Earth's first interstellar probe within a generation. Dr. Worden leads this Initiative as its Executive Director. 